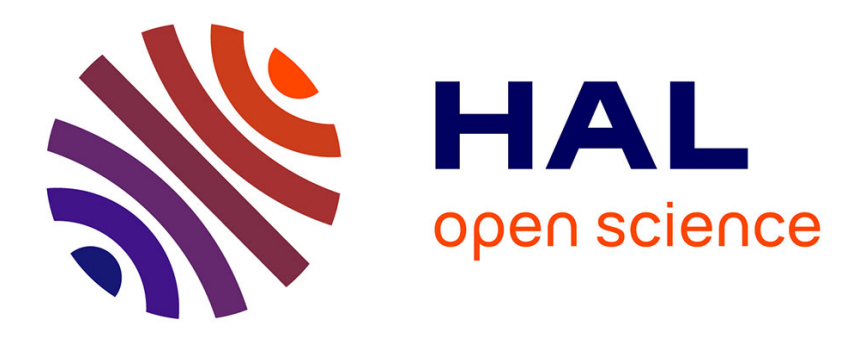

\title{
Mise en forme analytique de courbes et de surfaces fermées
}

\author{
J.P. Guillet, R. Amalric
}

\section{To cite this version:}

J.P. Guillet, R. Amalric. Mise en forme analytique de courbes et de surfaces fermées. Revue de Physique Appliquée, 1979, 14 (10), pp.903-905. 10.1051/rphysap:019790014010090300 . jpa00244677

\section{HAL Id: jpa-00244677 https://hal.science/jpa-00244677}

Submitted on 1 Jan 1979

HAL is a multi-disciplinary open access archive for the deposit and dissemination of scientific research documents, whether they are published or not. The documents may come from teaching and research institutions in France or abroad, or from public or private research centers.
L'archive ouverte pluridisciplinaire HAL, est destinée au dépôt et à la diffusion de documents scientifiques de niveau recherche, publiés ou non, émanant des établissements d'enseignement et de recherche français ou étrangers, des laboratoires publics ou privés. 


\title{
Mise en forme analytique de courbes et de surfaces fermées
}

\author{
J. P. Guillet $\left({ }^{*}\right)$, R. Amalric (**) \\ (*) Physicien, ESE \\ $\left({ }^{* *}\right)$ Chef du département des radiations de l'Institut J. Paoli-I. Calmettes, \\ 232 boulevard de Sainte-Marguerite, 13273 Marseille Cedex 2, France (Directeur : Pr. Y. Carcassonne)
}

(Reçu le 13 juin 1979, accepté le 16 juillet 1979)

\begin{abstract}
Résumé. - Les représentations de courbes ou de surfaces gauches par ensembles de coordonnées obtenues expérimentalement mobilisent un nombre élevé de données numériques. On propose une procédure basée sur la méthode des moindres carrés et minimisant le nombre final de données utiles. On donne des exemples simples et on suggère quelques autres applications possibles ainsi que des voies de développement de la méthode.
\end{abstract}

\begin{abstract}
Representation of plane curves or tridimensionnal surfaces by coordinates sets needs a high amount of numerical data. We propose a procedure based upon the least mean square method which minimizes the final number of useful data. We give some elementary examples and we suggest some other applications and ways of method developing.
\end{abstract}

L'étude de cette procédure a répondu initialement à un problème très spécifique : la représentation et l'archivage commode de courbes puis de surfaces isothermes fermées. Les mesures expérimentales fournissaient des ensembles de coordonnées affectées d'erreurs non systématiques. Il fallait obtenir une représentation simple qui inclue simultanément un lissage.

Quand on considère une courbe fermée, on peut, en l'exprimant en coordonnées polaires, l'assimiler à une courbe périodique, donc développable en série de Fourier. Cette procédure est difficilement applicable dans sa forme classique. En effet, elle implique des calculs d'intégrales requérant d'autant plus de temps qu'ils sont plus précis. D'autre part elle s'applique à des fonctions d'expression connue, ce qui n'est pas notre cas. Ces raisons ont fait choisir une méthode de moindres carrés. Soit l'expression d'une courbe plane fermée :

$$
r=\sum_{i=0}^{\infty}\left(a_{i} \cos i \theta+b_{i} \sin i \theta\right) .
$$

L'expression de la somme des erreurs quadratiques entre la courbe idéale et les $n$ points expérimentaux s'écrit :

$$
T=\sum_{k=1}^{n}\left[r\left(\theta_{k}\right)-r_{k}\right]^{2} \text {. }
$$

Sa minimisation donne les identités

$$
\frac{\partial T}{\partial a_{i}}=0 \quad \text { et } \quad \frac{\partial T}{\partial b_{i}}=0
$$

qui conduisent à un système d'équations linéaires dont les inconnues sont les coefficients cherchés :

$$
\begin{aligned}
& \sum_{k=1}^{n} \sum_{i=0}^{\infty}\left[\left(a_{i} \cos i \theta_{k}+b_{i} \sin i \theta_{k}-r_{k}\right) \cos l \theta_{k}\right]=0 \\
& \sum_{k=1}^{n} \sum_{i=0}^{\infty}\left[\left(a_{i} \cos i \theta_{k}+b_{i} \sin i \theta_{k}-r_{k}\right) \sin l \theta_{k}\right]=0 .
\end{aligned}
$$

Mathématiquement parlant, la restitution fidèle demanderait d'aller à l'harmonique infini. En fait on se limitera à un rang $p$ suffisant pour que les erreurs induites par la méthode soient négligeables devant celles dues à la mesure elle-même.

Pour une surface gauche fermée, on passe de une à deux variables. On peut étendre la méthode en supposant que la fonction exprimant cette surface peut se mettre sous la forme :

$$
\begin{gathered}
r=\sum_{\substack{i=0 \\
j=0}}^{\infty}\left(a_{i j} \cos i \theta \cos j z+b_{i j} \sin i \theta \cos j z+\cdots+\right. \\
\left.+c_{i j} \cos i \theta \sin j z+d_{i j} \sin i \theta \sin j z\right)
\end{gathered}
$$

Les variables prises ici correspondent à des coordonnées cylindriques mais pourraient être toute autre. Le système résultant de la minimisation des erreurs s'écrit :

$$
\sum_{k=1}^{n} \sum_{\substack{i=0 \\ j=0}}^{p}\left[\left[r\left(\theta_{k}, z_{k}\right)-r_{k}\right] \cos l \theta_{k} \cos m z_{k}\right]=0
$$




$$
\begin{aligned}
& \sum_{k=1}^{n} \sum_{\substack{i=0 \\
j=0}}^{p}\left[\left[r\left(\theta_{k}, z_{k}\right)-r_{k}\right] \sin l \theta_{k} \cos m z_{k}\right]=0 \\
& \sum_{k=1}^{n} \sum_{\substack{i=0 \\
j=0}}^{p}\left[\left[r\left(\theta_{k}, z_{k}\right)-r_{k}\right] \cos l \theta_{k} \sin m z_{k}\right]=0 \\
& \sum_{k=1}^{n} \sum_{\substack{i=0 \\
j=0}}^{p}\left[\left[r\left(\theta_{k}, z_{k}\right)-r_{k}\right] \sin l \theta_{k} \sin m z_{k}\right]=0
\end{aligned}
$$

avec $l$ et $m$ allant de 0 à $p$.

Dans la mesure du possible, on travaillera en harmoniques élevés et avec un grand nombre de points de mesure. Mais il y a des obstacles à la systématisation de cette règle, les plus importants concernant le temps de calcul et la capacité de l'ordinateur employé : on doit passer par une résolution matricielle. Compte tenu de l'élimination des lignes et colonnes nulles du fait de la nullité du coefficient $k$ pour les courbes et de $l$ et $m$ pour les surfaces, on devra réserver :

- Pour les courbes une matrice carrée de $(2 p+1)^{2}$ termes et deux tableaux de $2 p+1$ termes chacun.

- Pour les surfaces une matrice de $\left(4 p^{2}+4 p+1\right)^{2}$ termes et deux tableaux de $4 p^{2}+4 p+1$ termes chacun.

Il est alors relativement facile de traiter des courbes en harmoniques élevés compte tenu de la capacité des machines existantes; par contre, les surfaces requerront des outils plus puissants : l'harmonique 5 mobilise déjà 119064 octet.

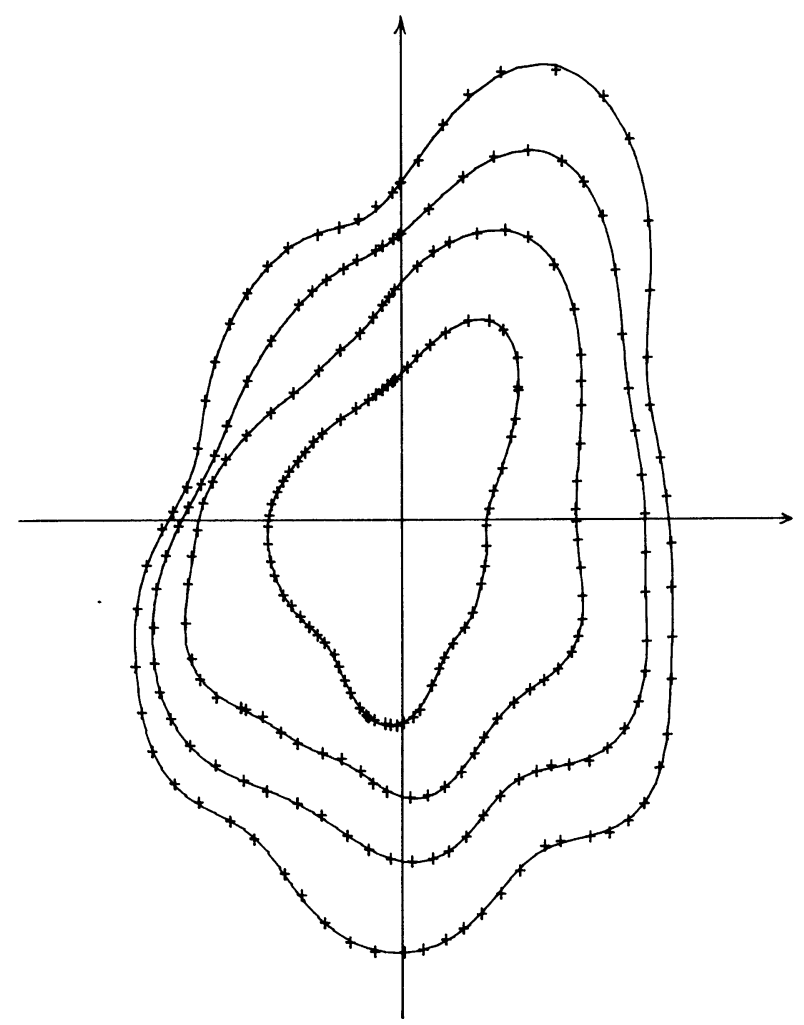

Fig. 1. - Représentation en harmonique 12 de courbes expérimentales.

[12th harmonic representation of an experimental curve.]
On donne à titre d'exemple les courbes de la figure 1 . Ce sont des isothermes purement expérimentales. A partir des coordonnées des points repérés par des croix, on a effectué une analyse en harmonique 12 d'où sont issus les coefficients qui ont permis de tracer les courbes en traits continus. La reproduction est de bonne qualité. Tout le réseau de courbes est alors stockable dans 4 tableaux de 25 coefficients chacun. Si l'on voulait mémoriser les coordonnées des points de mesure - environ 65 par courbe il faudrait 4 tableaux de 130 nombres chacun.

En montant en harmoniques élevés, on rencontre deux types de problèmes propres à cette méthode : en premier lieu, les procédures par moindres carrés tendent toujours à s'adapter le mieux possible aux points de base. Si on leur en donne l'occasion en montant en harmoniques, elles essaieront de faire passer la courbe résultante par tous les points, ce qui annulera le lissage. Ensuite, les longues séries de calculs associées aux hauts harmoniques conduisent à des cumuls d'erreurs du fait de la précision intrinsèque de calcul de l'ordinateur. On peut ainsi voir apparaître des coefficients d'un ordre de grandeur passablement erroné.

En conclusion, cette méthode peut permettre de notables économies de stockage de données. Il ne faut toutefois pas sous estimer ses défauts actuels :

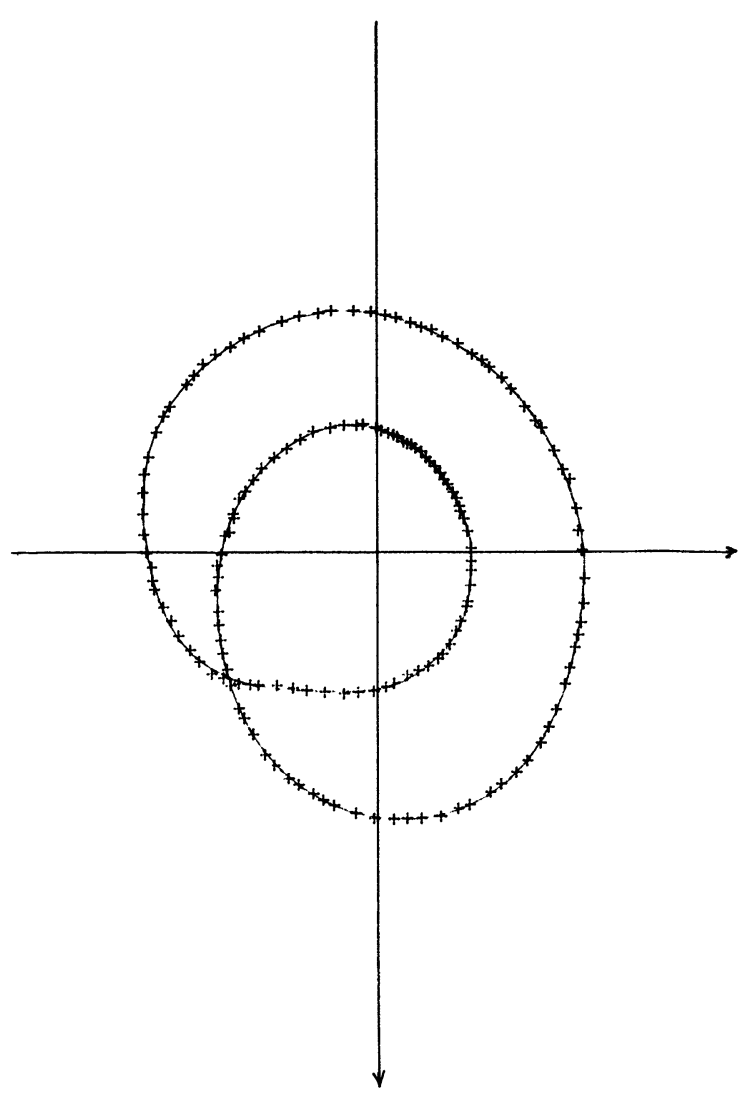

Fig. 2. - Représentation en harmonique 14 d'une courbe expérimentale à deux boucles imbriquées.

[14th harmonic representation of a two imbricated loops experimental curve.] 
- Pour des courbes comportant plusieurs boucles imbriquées, on ne pourra appliquer la méthode en question que si les boucles ont une surface intérieure commune ou au moins un point commun général. C'est le cas de la courbe de la figure 2.

- Une grande finesse de reproduction exige des harmoniques très élevés. $\mathrm{Si}$ on veut reproduire une tête humaine de $15 \mathrm{~cm}$ de diamètre en respectant les détails de $5 \mathrm{~mm}$ de largeur de base, il faudra prendre au moins $p=91$ ce qui conduit à 35721 coefficients. L'emploi de cette procédure en photogrammétrie exigerait des calculateurs puissants. En allant exagérément dans ce sens, si on souhaitait représenter le globe terrestre avec des détails de $100 \mathrm{~m}$ de base, il faudrait prendre $p=400000$ et inverser une matrice de $6,4 \times 10^{11}$ termes!

Toutefois, l'exemple de la figure 1 montre que l'on peut travailler sans trop de problèmes avec des courbes telles que celles obtenues dans nombre d'expérimentations courantes. Nos recherches porteront dans les mois à venir sur les problèmes de représentation des courbes à boucles ou à parties rentrantes ainsi que sur l'emploi d'autres bases - telles que $\sin x / x$ par exemple - qui permettraient d'optimiser la représentation. 